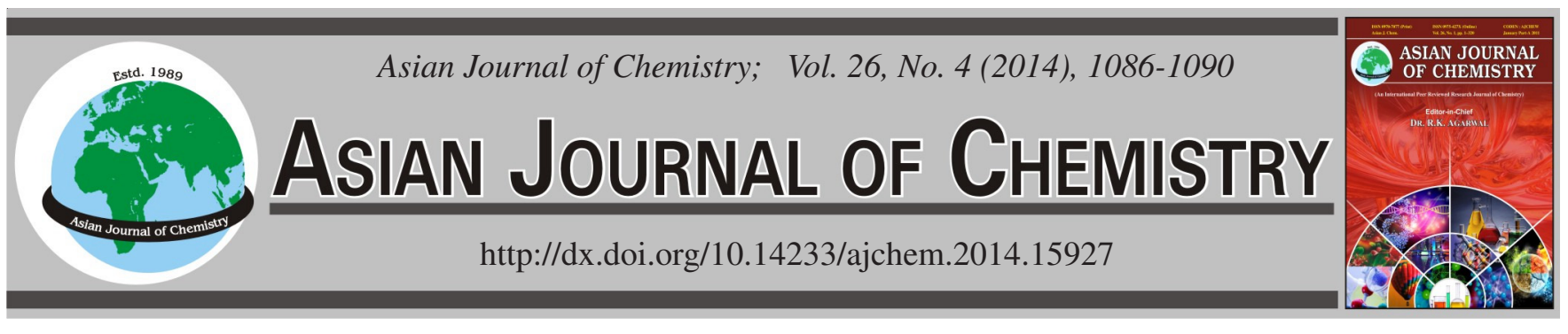

\title{
Phytochemical Investigations on Tribulus longipetalus
}

Muhammad Imran Anjum ${ }^{1,2}$, EJaz Ahmed ${ }^{3, *}$, Ahsan Sharif $^{3}$, Abdul Jabbar $^{1}$, Abdul Malik $^{4}$ and Nayab Batool ${ }^{3}$

\begin{abstract}
${ }^{1}$ Department of Chemistry, Baghdad-ul-Jadid Campus, The Islamia University Bahawalpur, Bahawalpur, Pakistan ${ }^{2}$ Post Graduate Department of Chemistry, Government SadiqEgerton College Bahawalpur, Bahawalpur, Pakistan ${ }^{3}$ Institute of Chemistry, University of the Punjab, Quaid-e-Azam Campus, Lahore-54590, Pakistan

${ }^{4}$ International Centre for Chemical and Biological Sciences, HEJ Research Institute of Chemistry, University of Karachi, Karachi-75270, Pakistan
\end{abstract}

*Corresponding author: E-mail: dr.ejaz.ahmed@gmail.com

The bioassay directed phytochemical investigations of Tribulus longipetalus have resulted the isolation of eighteen compounds and their structures havebeen elucidated by extensive use of modern spectroscopic (UV, IR, EIMS, HREIMS, ${ }^{1} \mathrm{H}$ NMR, ${ }^{13} \mathrm{C}$ NMR, FAB +ve, FAB -ve) techniques. All the compounds have been isolated for the first time from this species.

Keywords: Phytochemical, Tribulus longipetalus, Spectroscopy.

\section{INTRODUCTION}

Tribulus is very important genus of the family zygophyllaceae and it grows as shrubs in tropical and subtropical regions throughout the world. The genus Tribulus comprises about 20 species and represented by 4 species in Pakistan ${ }^{1}$. Tribulus longipetalus, locally known as bhakri, is a prostrate, green or grayish-white in colour. Its leaves are 1-4 cm long and flowers are mostly yellow in colour. The fruit of this plant is broad and somewhat pointed. The plant possesses cooling, demulcent, diuretic, tonic aphrodisiacs aperients properties and its fruit is used in urinary disorders, impotence, heart diseases. The seeds of $T$. longipetalus are known to be used in diseases of kidney stone, gout and hemorrhages ${ }^{2}$. Tribulus longipetalus has a syn. name Tribulus alatus. No literature is available with the name Tribulus longipetalus, but a little bit work has been reported for the Tribulus alatus. Literature survey revealed that only three steroidal saponins from the $\mathrm{MeOH}$ extract of the aerial parts of the Tribulusalatus Del. have so far been reported $^{3}$. In the present study eighteen compounds have been isolated for the first time from the methanolic extract of the whole plant of $T$. longipetalus. They were identified as 1triacontanol $^{4}(\mathbf{1})$, hexacosanoic $\operatorname{acid}^{5}(\mathbf{2})$, tetracosanoic acid 6 (3), benzoic $\operatorname{acid}^{7}(4), 2$-hydroxybenzoic $\operatorname{acid}^{8}(5)$, 4-ethoxy benzoic $\operatorname{acid}^{9}(6), 3,5$-dimethoxybenzoic $\operatorname{acid}^{10}(7), \beta$ -

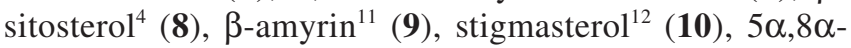
epidioxyergosta-6,9(11),22-trien-3 $\beta$-ol ${ }^{13}(\mathbf{1 1})$, ursolic acid ${ }^{4,10}$ (12), cyclostenol $^{14}(\mathbf{1 3})$, oleanolic acid ${ }^{15}(\mathbf{1 5})$, 5,6-dihydroxy- 4,7-dimethoxy-2-methylanthracene-9,10-dione ${ }^{16}(\mathbf{1 5}), 1,3-$ dihydroxycyclolanosterol $^{17}$ (16), stigmasterol 3-O- $\beta$-Dglucopyranoside ${ }^{15}(\mathbf{1 7})$ and $\beta$-sitosterol 3-O- $\beta$-D-glucopyranoside $^{18}$ (18), (Fig. 1).

\section{EXPERIMENTAL}

UV and IR spectra were recorded on Hitachi-UV-3200 and Jasco-320-A spectrometers, respectively. ${ }^{1} \mathrm{H}$ and ${ }^{13} \mathrm{C}$ NMR spectra were recorded on a Bruker AM-400 spectrometer with tetramethylsilane (TMS) as an internal standard. The 2D-NMR spectra were recorded on a Bruker AMX 500 NMR spectrometer. Optical rotations were measured on a Jasco DIP-360 digital polarimeter using a $10 \mathrm{~cm}$ tube. Mass spectra (EIMS and HR-EIMS, FAB-MS) were measured in an electron impact mode on finnigan MAT 12 and MAT 312 spectrometers and ions were given in $m / z(\%)$. TLC was performed on precoated silica gel F254 plates, the detection was done at $254 \mathrm{~nm}$ and by spraying with ceric sulphate reagent. Silica gel (E. Merck, 230-400 mesh) was used for column chromatography. Melting points were determined on a gallenkemp apparatus and are uncorrected.

The whole plant of Tribulus longipetalus Viv. was collected from Cholistan Desert near Bahawalpur (Pakistan) in April 2008 and identified by Dr. Muhammad Arshad, Plant Taxonomist Cholistan Institute for Desert Studies (CIDS), Islamia University Bahawalpur, Bahawalpur, Pakistan, where a voucher specimen has been deposited. 

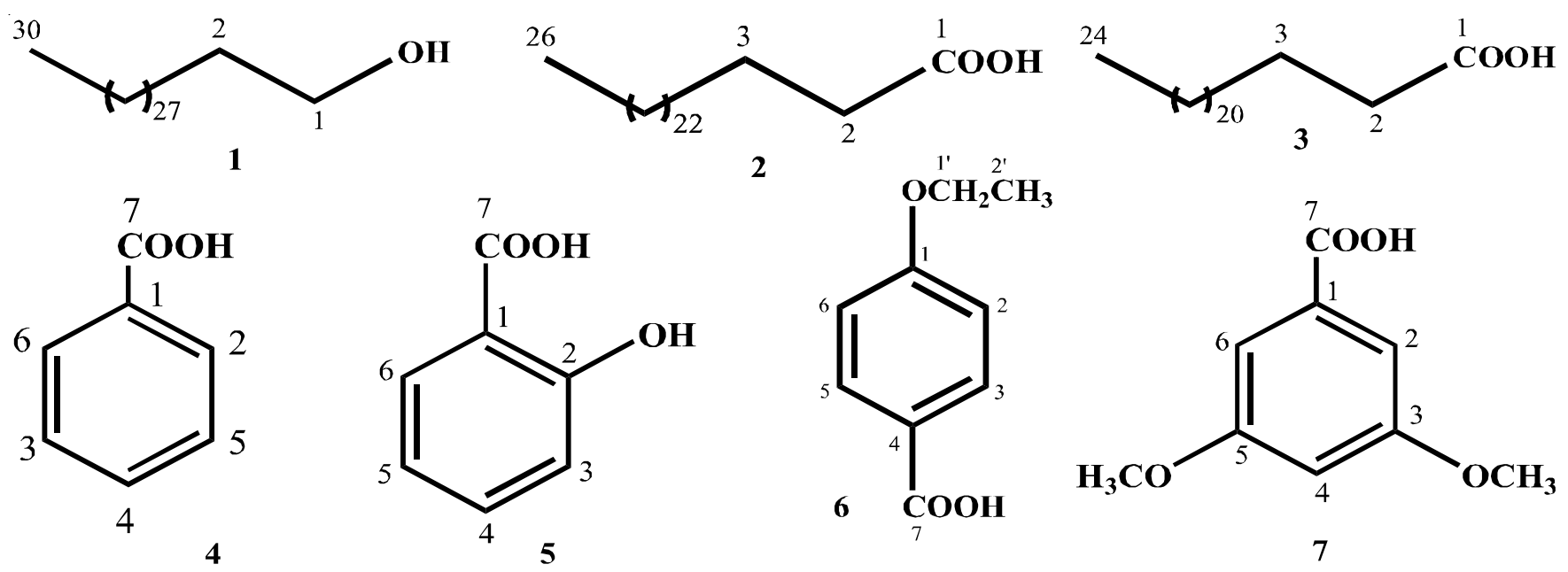

$2 \quad \mathrm{OCH}_{2}^{\prime \prime}{ }^{2 !} \mathrm{H}_{3}$
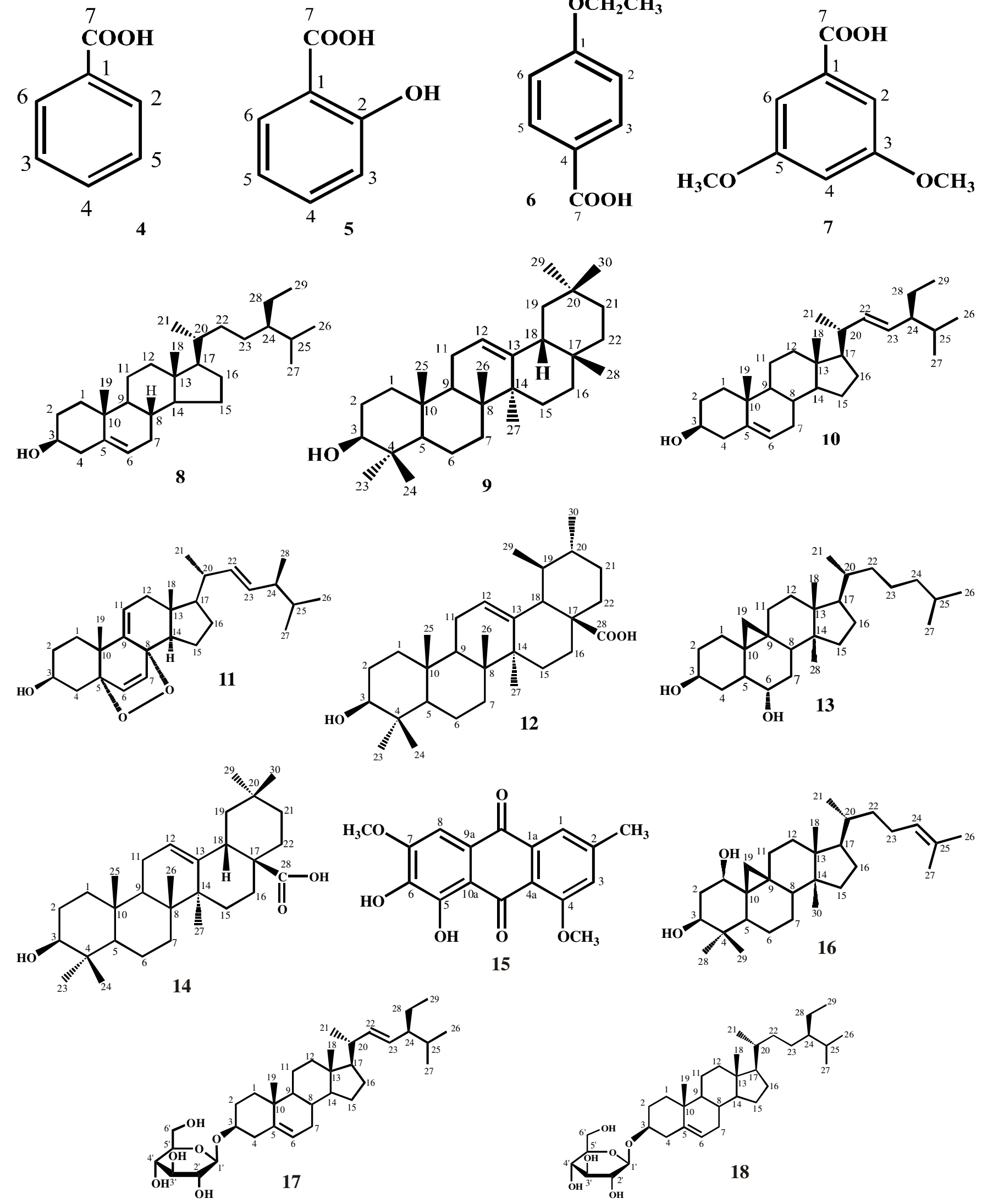

Fig. 1. Compounds isolated from T. longipetalus 
Extraction and isolation: The air dried whole plant (10 $\mathrm{Kg}$ ) of Tribulus longipetalus were powdered and extracted at room temperature with methanol $(3 \times 15 \mathrm{~L})$. The extract was concentrated under reduced pressure to yield residue $800 \mathrm{~g}$, partitioned with $\mathrm{H}_{2} \mathrm{O}$ and $n$-hexane. The $n$-hexane soluble portion was concentrated with rotary evaporator and obtained greenish material $150 \mathrm{~g}$ amount. The crude extract was again partitioned between $\mathrm{CHCl}_{3}(180 \mathrm{~g})$, ethyl acetate $(50 \mathrm{~g}), n$ butanol $(140 \mathrm{~g})$ and $\mathrm{H}_{2} \mathrm{O}(50 \mathrm{~g})$ soluble fractions. The $\mathrm{CHCl}_{3}$ soluble fraction (180 g) was subjected to column chromatography over silica gel eluting with $n$-hexane- $\mathrm{CHCl}_{3}, \mathrm{CHCl}_{3}$ and $\mathrm{CHCl}_{3}-\mathrm{MeOH}$ in increasing order of polarity, to obtain 15 fractions (T1-T15). The fraction obtained from $n$-hexane$\mathrm{CHCl}_{3}$ (8.0: 2.0) labeled as T2 was again chromatographed over silica gel eluting with same solvent system gave three fractions A1-A3. The fraction A2 obtained from $n$-hexane$\mathrm{CHCl}_{3}$ (8.5: 1.5) produced three spots on TLC which were again purified by PTLC using the solvent system $n$-hexaneEtOAC (8.8:1.2), provided benzoic acid (4), 2-hydroxy benzoic acid (5) and 4-ethoxy benzoic acid (6). The fraction A3 was purified over silica gel column chromatography by using solvent system $n$-hexane-EtOAC (9.0: 1.0) gave 3,5-dimethoxy benzoic acid (7). The fractions obtained from $n$-hexane- $\mathrm{CHCl}_{3}$ (7.0: 3.0) gave five spots on TLC, were combined and rechromatographed over silica gel column chromatography using the solvent system $n$-hexane-EtOAC $(8.5: 1.5)$ produced three spots on TLC which were combined and purified on PTLC by using the same solvent system gave $\beta$-sitosterol (8), $\beta$-amyrine (9) and cyclostenol (13), respectively. The portion got from $n$ hexane-EtOAC (8.3:1.7) were combined and then rechromatographed over the silica gel column chromatography by using solvent system $n$-hexane-EtOAC $(8.5: 1.5)$ gave two pure compounds stigmasterol (10) and 1-triacontanol (1). The portions which were obtained from $n$-hexane- $\mathrm{CHCl}_{3}$ (5.0: 5.0) showed many spots on TLC, again purified by repeated silica gel column chromatography to give four fractions (A-D). The fraction $\mathrm{A}$ ( $n$-hexane- $\left.\mathrm{CHCl}_{3}, 6.6: 3.4\right)$ was again purified by the silica gel column chromatography using same solvent system to provide tetracosanoic acid (3) and $5 \alpha, 8 \alpha$-epidioxyergosta 6,9(11), 22-triene-3- $\beta$-ol (11). The fractions B and C were separately treated with the silica gel column chromatography by using the same solvent system $n$-hexane- $\mathrm{CHCl}_{3}(6.5$ : 3.5) and (6.4: 3.6) gave ursolic acid (12) and hexacosanoic acid (2), respectively. The fraction D was treated with PTLC using the solvent system $n$-hexane- $\mathrm{CHCl}_{3}$ in the ratio (6.0:4.0) gave 1,3-dihydroxycyclolanosterol (16) and oleanolic acid (14). The fraction obtained from $n$-hexane- $\mathrm{CHCl}_{3}$ (2.0: 8.0) gave many spots on TLC, which on PTLC using solvent system $n$-hexane- $\mathrm{CHCl}_{3}$ (3.0: 7.0 ) provided 5,6-dihydroxy 4,7dimethoxy-2-methyl anthracene 9,10-dione (15) as a major compound while the other compounds were unable to isolate by the available chromatographic techniques. The fractions which were obtained from $\mathrm{CHCl}_{3}-\mathrm{MeOH}$ (1.5: 8.5) were combined and rechromatographed over silica gel eluting with $\mathrm{CHCl}_{3}-\mathrm{MeOH}$ provided three fractions. The fraction 2 which was obtained from $\mathrm{CHCl}_{3}-\mathrm{MeOH}$ (1.7: 8.3) gave two spots on TLC, which on PTLC by using same solvent system afforded stigmasterol-3-O- $\beta$-D-glucopyranoside (17) and $\beta$-sitosterol3-O- $\beta$-D-glucopyranoside $(\mathbf{1 8})$, respectively.
1-Triacontanol (1): Colorless crystals, $41 \mathrm{mg}$, m.p. 86$87^{\circ} \mathrm{C}$. IR (KBr, $\left.v_{\max }, \mathrm{cm}^{-1}\right)$ : 3520, 3060, 810. EIMS: $\mathrm{m} / z$ (rel. int.) $[\mathrm{M}]^{+}$438, 420, 405, 360. HR-EIMS $m / z 438.4860$ (calcd. for $\left.\mathrm{C}_{30} \mathrm{H}_{62} \mathrm{O}, 438.4801\right)$. ${ }^{1} \mathrm{H}$ NMR $\left(\mathrm{CDCl}_{3}, 400 \mathrm{MHz}\right) \delta: 3.14$ $(2 \mathrm{H}, \mathrm{t}, J=7.1 \mathrm{~Hz}, \mathrm{H}-1), 1.61$ (2H, m, H-2), 1.62-1.29 (54 H, br. s, H-3-H-29), 0.91 (3H, t, $J=6.7 \mathrm{~Hz}, \mathrm{H}-30) .{ }^{13} \mathrm{C}$ NMR $\left(\mathrm{CDCl}_{3}, 100 \mathrm{MHz}\right) \delta$ : 63.0 (C-1), 32.02 (C-2), 30.0 (C-3), 29.8 (C-4), 29.4-28.8, (C-5-C-28), 21.2 (C-29), 13.7 (C-30).

Hexacosanoic acid (2): White crystals, 38 mg, m.p. 88$89^{\circ} \mathrm{C}$. IR $\left(\mathrm{KBr}, \mathrm{v}_{\max }, \mathrm{cm}^{-1}\right): 3280-2620,1716,920$. EIMS: $\mathrm{m} / z$ (rel. int.): $[\mathrm{M}]^{+} 396$ (58), 378 (100), 363 (41), 318, 303. HREIMS $m / z 396.3901$ (calcd. for $\left.\mathrm{C}_{26} \mathrm{H}_{52} \mathrm{O}_{2}, 396.3967\right)$. ${ }^{1} \mathrm{H}$ NMR $\left(\mathrm{CDCl}_{3}, 400 \mathrm{MHz}\right) \delta: 10.57(\mathrm{OH}), 2.26(2 \mathrm{H}, \mathrm{t}, J=7.4 \mathrm{~Hz})$ $1.59(2 \mathrm{H}, \mathrm{m}) 1.29-1.18\left(48 \mathrm{H}, \mathrm{br}, \mathrm{s}, 24 \times \mathrm{CH}_{2}\right) 0.90(3 \mathrm{H}, \mathrm{t}, J=$ $\left.6.4 \mathrm{~Hz}, \mathrm{CH}_{3}\right) .{ }^{13} \mathrm{C} \mathrm{NMR}\left(\mathrm{CDCl}_{3}, 100 \mathrm{MHz}\right) \delta: 181.0(\mathrm{C}-1)$ 33.9 (C-2) 29.9 (C-3) 29.7-29.2 (C-4-C-25), 13.7 (C-26).

Tetracosanoic acid (3): Colorless needles, 22 mg. m.p. 87-88 ${ }^{\circ} \mathrm{C}$. IR $\left(\mathrm{KBr}, \mathrm{v}_{\max }, \mathrm{cm}^{-1}\right): 3280-2610,1710,910$. EIMS $\mathrm{m} / z$ (rel. int.): $[\mathrm{M}]^{+} 368$ (60), 350 (100), 335 (55), 290 (40). HR-EIMS m/z 368.3605 (calcd. for $\mathrm{C}_{24} \mathrm{H}_{48} \mathrm{O}_{2}, 368.3654$ ). ${ }^{1} \mathrm{H}$ NMR $\left(\mathrm{CDCl}_{3}, 400 \mathrm{MHz}\right) \delta: 10.47(\mathrm{COO} \underline{\mathrm{H}}), 2.21(2 \mathrm{H}, \mathrm{t}, \mathrm{J}$ $=7.4 \mathrm{~Hz}, \mathrm{H}-2), 1.57$ (2H, m, H-3), 1.29-1.17 (40 H, br s, $20 \times$ $\left.\mathrm{CH}_{2}, \mathrm{H}-4-\mathrm{H}-23\right), 0.90$ (3H, t, $\left.J=6.4 \mathrm{~Hz}, \mathrm{H}-24\right) .{ }^{13} \mathrm{C}$ NMR $\left(\mathrm{CDCl}_{3}, 100 \mathrm{MHz}\right) \delta: 180.3$ (C-1) 34.2 (C-2) 29.6 (C-3) 29.229.0 (C-4-C-23), 13.7 (C-24).

Benzoic acid (4): White crystals, $28 \mathrm{mg}$, m.p. 122-123 ${ }^{\circ} \mathrm{C}$. UV $\left(\mathrm{CD}_{3} \mathrm{OD}\right) \lambda_{\max } \log \varepsilon: 228$ (3.62), 272 (3.77), 300 (4.01) nm. HREIMS $m / z$ (rel. int.): $[\mathrm{M}]^{+} 122.0367$ (calcd. for $\mathrm{C}_{7} \mathrm{H}_{5} \mathrm{O}_{2}$, 122.0360). IR (KBr, $\left.v_{\max }, \mathrm{cm}^{-1}\right)$ : 3260-2610 (COOH), 1705 $(\mathrm{C}=\mathrm{O}) .{ }^{1} \mathrm{H}$ NMR $\left(\mathrm{CDCl}_{3}, 400 \mathrm{MHz}\right) \delta: 11.92(1 \mathrm{H}, \mathrm{s}, \mathrm{O}-\mathrm{H})$, $8.12(2 \mathrm{H}, \mathrm{t}, J=8.5 \mathrm{~Hz}, \mathrm{H}-2$ and $\mathrm{H}-6), 7.60(1 \mathrm{H}, \mathrm{t}, J=8.5 \mathrm{~Hz}$, $\mathrm{H}-4), 7.46(2 \mathrm{H}, \mathrm{t}, J=8.5 \mathrm{~Hz}, \mathrm{H}-3$ and $\mathrm{H}-5) .{ }^{13} \mathrm{C} \mathrm{NMR}\left(\mathrm{CD}_{3} \mathrm{OD}\right.$, $100 \mathrm{MHz})$ 8: 180.0 (C-7), 134.5 (C-4), 131.1 (C-1), 130.7 (C2 and C-6), 130.7 (C-3 and C-5).

2-Hydroxybenzoic acid (5): White crystals, $18 \mathrm{mg}$, m.p. 159-160 ${ }^{\circ} \mathrm{C}$. UV $(\mathrm{MeOH}) \lambda_{\max } \mathrm{nm}(\log \varepsilon): 234$ (3.67), 302 (3.98). IR $\left(\mathrm{CHCl}_{3}, \mathrm{v}_{\max }, \mathrm{cm}^{-1}\right): 3408,3246,2657,1704,1626$, 812. EIMS $m / z$ (rel. int.): [M] $]^{+} 138$ (93), 120 (100), 92 (94), 81 (27), 64 (65), 53 (61). HREIMS m/z 138.0291 (calcd. for $\left.\mathrm{C}_{7} \mathrm{H}_{6} \mathrm{O}_{3}, 138.0316\right) .{ }^{1} \mathrm{H}$ NMR $\left(\mathrm{CDCl}_{3}, 400 \mathrm{MHz}\right) \delta: 7.84(1 \mathrm{H}$, $\delta, J=8.5 \mathrm{~Hz}, \mathrm{H}-6), 7.44(1 \mathrm{H}, \mathrm{t}, J=8.5 \mathrm{~Hz}, \mathrm{H}-4), 6.89(1 \mathrm{H}, \mathrm{t}$, $J=8.5 \mathrm{~Hz}, \mathrm{H}-5)$ and $6.85(1 \mathrm{H}, \mathrm{d}, J=8.5 \mathrm{~Hz}, \mathrm{H}-3) .{ }^{13} \mathrm{C} \mathrm{NMR}$ $\left(\mathrm{CDCl}_{3}, 100 \mathrm{MHz}\right) \delta$ : 172.9 (C-7), 162.5 (C-2), 136.6 (C-4), 131.1 (C-6), 119.8 (C-5), 117.8 (C-3) 112.4 (H-1).

4-Ethoxy benzoic acid (6): White crystals, $25 \mathrm{mg}$, m.p. 195-196 ${ }^{\circ} \mathrm{C}$. IR $\left(\mathrm{CHCl}_{3}, v_{\max }, \mathrm{cm}^{-1}\right): 3080,2400,1715,1610$ 1540. EIMS m/z (rel. int.): [M] 166 (10), 137 (50), 120 (100). HREIMS $m / z$ : 166.0615 (calcd. for $\mathrm{C}_{9} \mathrm{H}_{10} \mathrm{O}_{3}, 166.0630$ ). ${ }^{1} \mathrm{H}$ NMR $\left(\mathrm{CDCl}_{3}, 500 \mathrm{MHz}\right) \delta: 6.85(2 \mathrm{H}, \mathrm{dd}, J=7.8,1.6 \mathrm{~Hz}$, H-2 and H-6), 7.95 (2H, dd, $J=7.8,2.2 \mathrm{~Hz}, \mathrm{H}-3$ and H-5), $4.20\left(2 \mathrm{H}, \mathrm{q}, J=4.9 \mathrm{~Hz}, \mathrm{OCH}_{2} \mathrm{CH}_{3}\right)$ and $1.10(3 \mathrm{H}, \mathrm{t}, J=6.8$ $\left.\mathrm{Hz}, \mathrm{OCH}_{2} \mathrm{CH}_{3}\right) .{ }^{13} \mathrm{C} \mathrm{NMR}\left(\mathrm{CDCl}_{3}, 125 \mathrm{MHz}\right) \delta: 122.6(\mathrm{C}-1)$, 132.1 (C-2, C-6), 115.4 (C-3, C-5), 160.3 (C-4), 66.2 $\left(\mathrm{OCH}_{2} \mathrm{CH}_{3}\right), 20.1\left(\mathrm{OCH}_{2} \mathrm{CH}_{3}\right)$ and $167.1(\mathrm{CO})$.

3,5-Dimethoxybenzoic acid (7): Colorless crystals, 20 mg m.p. $182.5^{\circ} \mathrm{C}$. UV (MeOH) $\lambda_{\max } \mathrm{nm}(\log \varepsilon): 220,251,285$ $\mathrm{nm}$. IR $\left(\mathrm{KBr}, v_{\max }, \mathrm{cm}^{-1}\right): 3550(\mathrm{OH}), 1710(\mathrm{C}=\mathrm{O}), 1625$ (aromatic). EIMS $m / z$ (rel. int. \%): [M] $]^{+} 182$ (88), 151 (45), 120 (100), 92 (70), 64 (52), 53 (80). HREIMS m/z: 182.1739 (calcd. for 
$\left.\mathrm{C}_{9} \mathrm{H}_{10} \mathrm{O}_{4}, 182.1706\right) .{ }^{1} \mathrm{H}$ NMR $\left(\mathrm{CD}_{3} \mathrm{OD}, 400 \mathrm{MHz}\right) \delta: 7.09(2 \mathrm{H}$, $\mathrm{d}, J=2.0 \mathrm{~Hz}, \mathrm{H}-2$ and $\mathrm{H}-6), 6.72(1 \mathrm{H}, \mathrm{d}, J=2.1 \mathrm{~Hz}, \mathrm{H}-4)$, $3.82\left(6 \mathrm{H}, \mathrm{s}, 2 \times \mathrm{OCH}_{3}\right) .{ }^{13} \mathrm{C} \mathrm{NMR}\left(\mathrm{CD}_{3} \mathrm{OD}, 100 \mathrm{MHz}\right) \delta: 167.5$ $(\mathrm{COOH}), 161.5$ (C-3 and C-5), 130.7 (C-1), 105.2 (C-2 and C-6), 101.3 (C-4)

$\boldsymbol{\beta}$-Sitosterol (8): Crystallized from acetone, $25 \mathrm{mg}$ m.p. $135^{\circ} \mathrm{C},[\alpha]_{\mathrm{D}}^{27}+35.5^{\circ}\left(\mathrm{c}=1.02, \mathrm{CHCl}_{3}\right)$. IR $\left(\mathrm{KBr}, v_{\max }, \mathrm{cm}^{-1}\right)$ : 3446, 3050, 1650. EIMS m/z (rel. int. \%): [M] 414 (15), 399 (10), 396 (12), 381 (72), 329 (28), 275 (12), 273 (17), 255 (36). HREIMS $m / z$ : 414.3845 (calcd. for $\mathrm{C}_{29} \mathrm{H}_{50} \mathrm{O}, 414.3861$ ). ${ }^{1} \mathrm{H}$ NMR $\left(\mathrm{CDCl}_{3}, 400 \mathrm{MHz}\right) \delta: 5.11(1 \mathrm{H}, \mathrm{m}, \mathrm{H}-6), 3.36(1 \mathrm{H}$, m, H-3), 1.01 (3H, s, Me-19), $0.92(3 \mathrm{H}, \mathrm{d}, J=6.2 \mathrm{~Hz}, \mathrm{Me}-$ 21), $0.84(3 \mathrm{H}, \mathrm{t}, J=7.0 \mathrm{~Hz}, \mathrm{Me}-29), 0.83(3 \mathrm{H}, \mathrm{d}, J=6.5 \mathrm{~Hz}$, Me-26), 0.81 (3H, d, $J=6.5 \mathrm{~Hz}, \mathrm{Me}-27), 0.68$ (3H, s, Me-18). ${ }^{13} \mathrm{C} \mathrm{NMR}\left(\mathrm{CDCl}_{3}, 100 \mathrm{MHz}\right) \delta: 140.9$ (C-5), 121.9 (C-6), 71.9 (C-3), 56.8 (C-14), 56.2 (C-17), 50.8 (C-9), 50.4 (C-24), 42.6 (C-13), 42.4 (C-4), 40.3 (C-12), 37.3 (C-1), 36.6 (C-10), 36.3 (C-20), 34.0 (C-22), 32.3 (C-7), 32.0 (C-8), 31.8 (C-2), 29.3 (C-23), 28.2 (C-16), 26.2 (C-25), 24.3 (C-15), 23.1 (C-28), 21.1 (C-11), 19.8 (C-27), 19.4 (C-19), 19.1 (C-21), 18.8 (C26), 11.9 (C-29), 11.4 (C-18).

$\boldsymbol{\beta}$-Amyrin (9): Crystallized from ethanol, $30 \mathrm{mg}$, m.p. $197-198{ }^{\circ} \mathrm{C},[\alpha]_{\mathrm{D}}^{25}+100^{\circ}\left(\mathrm{c}=1.15, \mathrm{CHCl}_{3}\right)$. IR $\left(\mathrm{KBr}, \mathrm{v}_{\max }\right.$, $\mathrm{cm}^{-1}$ ): 3510, 3055, 1635, 820. EIMS $\mathrm{m} / z$ (rel. int. \%) $[\mathrm{M}]^{+}$: 426 (15), 411 (18), 408 (16), 393 (32), 257 (20), 218 (100), 207 (10), 203 (40) 189 (55). HREIMS m/z 426.3825 (calcd. for $\left.\mathrm{C}_{30} \mathrm{H}_{50} \mathrm{O}, 426.3861\right) .{ }^{1} \mathrm{H} \mathrm{NMR}\left(\mathrm{CDCl}_{3}, 500 \mathrm{MHz}\right) \delta: 5.11$ (1H, m, H-12), 3.19 (1H, dd, $J=10.0,4.5 \mathrm{~Hz}, \mathrm{H}-3), 1.08(3 \mathrm{H}$, s, Me-27), 1.02 (3H, s, Me-23), 1.01 (3H, s, Me-24), 0.96 (3H, s, Me-25), 0.93 (3H, s, Me-29), 0.88 (3H, s, Me-26), 0.85 (3H, s, Me-30) and $0.80(3 \mathrm{H}, \mathrm{s}, \mathrm{Me}-28) .{ }^{13} \mathrm{C} \mathrm{NMR}\left(\mathrm{CDCl}_{3}\right.$, $125 \mathrm{MHz}$ ) $\delta: 144.3$ (C-13), 124.1 (C-12), 78.8 (C-3), 54.4 (C5), 47.7 (C-9), 47.3 (C-18), 46.9 (C-19), 42.3 ( C-14), 41.6 (C-22), 40.9 (C-8), 39.4 (C-4), 39.0 (C-1), 37.0 (C-10), 34.0 (C-17), 33.3 (C-29), 33.2 (C-7), 32.9 (C-21), 31.7 (C-20), 28.2 (C-23), 28.0 (C-28), 27.4 (C-2), 26.5 (C-16), 26.3 (C-15), 26.0 (C-27), 23.6 (C-11), 23.3 (C-30), 18.5 (C-6), 16.9 (C-26), 15.6 (C-25), 15.1 (C-24).

Stigmasterol (10): White crystals, 25 mg, m.p. 170$171{ }^{\circ} \mathrm{C} .[\alpha]_{\mathrm{D}}{ }^{25} 51.5^{\circ}\left(\mathrm{c}=1.06, \mathrm{CHCl}_{3}\right)$. IR $\left(\mathrm{CHCl}_{3}, \mathrm{v}_{\max }, \mathrm{cm}^{-1}\right)$ : $3432(\mathrm{OH}), 1648(\mathrm{C}=\mathrm{C})$. EIMS: $m / z$ (rel. int. \%): $[\mathrm{M}]^{+} 412$ (8), 396 (12), 394 (20), 379 (27), 369 (35), 351 (71), 327 (60), 301 (18), 300 (67), 273 (30), 270 (24). HREIMS m/z: 412.3920 (calcd. for $\left.\mathrm{C}_{29} \mathrm{H}_{48} \mathrm{O}, 412.3926\right) .{ }^{1} \mathrm{H} \mathrm{NMR}\left(\mathrm{CDCl}_{3}, 400 \mathrm{MHz}\right)$ $\delta: 5.33$ (1H, m, H-6), 5.15 (1H, dd, $J=15.2,8.4 \mathrm{~Hz}, \mathrm{H}-22)$, $5.02(1 \mathrm{H}, \mathrm{dd}, J=15.2,8.6 \mathrm{~Hz}, \mathrm{H}-23), 3.28$ (1H, m, H-3), 0.90 $(3 \mathrm{H}, \mathrm{d}, J=6.5 \mathrm{~Hz}, \mathrm{Me}-21), 0.83(3 \mathrm{H}, \mathrm{d}, J=6.6 \mathrm{~Hz}, \mathrm{Me}-26)$, $0.84(3 \mathrm{H}, \mathrm{t}, J=7.0 \mathrm{~Hz}, \mathrm{Me}-29), 0.81(3 \mathrm{H}, \mathrm{d}, J=6.5 \mathrm{~Hz}, \mathrm{Me}-$ 27), 0.80 (3H, s, Me-19), 0.65 (3H, s, Me-18). ${ }^{13} \mathrm{C}$ NMR $\left(\mathrm{CDCl}_{3}, 100 \mathrm{MHz}\right)$ \&: 140.9 (C-5), 138.4 (C-22), 129.4 (C23), 121.7 ( C-6), 71.9 (C-3), 57.0 (C-14), 56.0 (C-17), 51.3 (C-24), 50.3 (C-9), 42.5 (C-13), 42.2 (C-4), 40.5 (C-20), 39.7 (C-12), 37.5 (C-1), 36.6 (C-10), 32.2 (C-8), 32.0 (C-25), 31.9 (C-7), 31.4 (C-2), 28.9 (C-16), 25.4 (C-28), 24.4 (C-15), 21.6 (C-27), 21.3 (C-21), 21.0 (C-11), 19.4 (C-19), 19.0 (C-26), 12.4 (C-18), 12.0 (C-29).

$5 \alpha, 8 \alpha$-Epidioxyergosta-6,9(11),22-trien-3 $\beta$-ol (11): White amorphoussolid, $15 \mathrm{mg},[\alpha]_{\mathrm{D}}{ }^{25}-33.0^{\circ} \mathrm{C}(\mathrm{c}=1.00$, $\mathrm{CHCl}_{3}$ ). IR (KBr, $\left.v_{\max }, \mathrm{cm}^{-1}\right): 3440,3024$ and 1640. EIMS: $\mathrm{m} / \mathrm{z}$ (rel. int.): [M] 426 (28), 410 (35), 394 (100), 376 (15), 251 (38). HREIMS m/z: 426.641 (calcd. for $\mathrm{C}_{28} \mathrm{H}_{42} \mathrm{O}_{3}, 426.638$ ). ${ }^{1} \mathrm{H} \mathrm{NMR}\left(\mathrm{CDCl}_{3}, 500 \mathrm{MHz}\right) \delta: 6.61(1 \mathrm{H}, \mathrm{d}, J=8.5 \mathrm{~Hz}, \mathrm{H}-7)$, $6.30(1 \mathrm{H}, \mathrm{d}, J=8.5 \mathrm{~Hz}, \mathrm{H}-6), 5.43(1 \mathrm{H}, \mathrm{dd}, J=6.0,1.8 \mathrm{~Hz}, \mathrm{H}-$ 11), 5.24 (1H, dd, $J=15.1,8.0 \mathrm{~Hz}, \mathrm{H}-22), 5.17(1 \mathrm{H}, \mathrm{dd}, J=$ 15.1, 8.5Hz, H-23), 3.98 (1H, m, H-3), 1.09 (3H, s, H-19), $1.01(3 \mathrm{H}, \mathrm{d}, J=6.6 \mathrm{~Hz}, \mathrm{H}-21), 0.92(3 \mathrm{H}, \mathrm{d}, J=7.2 \mathrm{~Hz}, \mathrm{H}-28)$, $0.84(3 \mathrm{H}, \mathrm{d}, J=6.7 \mathrm{~Hz}, \mathrm{H}-27), 0.83(3 \mathrm{H}, \mathrm{d}, J=6.5 \mathrm{~Hz}, \mathrm{H}-26)$ and $0.75(3 \mathrm{H}, \mathrm{s}, \mathrm{H}-18) .{ }^{13} \mathrm{C} \mathrm{NMR}\left(\mathrm{CDCl}_{3}, 125 \mathrm{MHz}\right) \delta: 142.8$ (C-9), 135.8 (C-7), 135.5 (C-22), 132.5 (C-23), 135.2 (C-6), 119.8 (C-11), 83.0 (C-5), 78.5 (C-8), 66.4 (C-3), 56.0 (C-17), 48.3 (C-14), 43.7 (C-13), 42.8 (C-24), 41.3 (C-12), 39.9 (C20), 38.1 (C-10), 36.2 (C-4), 33.1 (C-25), 32.8 (C-1), 30.8 (C2), 28.7 (C-16), 25.5 (C-19), 22.8 (C-15), 20.9 (C-21), 20.0 (C-27), 19.7 (C-26), 17.5 (C-28) 12.8 (C-18).

Ursolic acid (12): Colorless needles, 25 mg, m.p. 283$285^{\circ} \mathrm{C},[\alpha]_{\mathrm{D}}{ }^{28}+62.5^{\circ}\left(\mathrm{c}=1.00, \mathrm{CHCl}_{3}\right) . \mathrm{IR}\left(\mathrm{CHCl}_{3}, \mathrm{v}_{\max }\right.$, $\left.\mathrm{cm}^{-1}\right): 3510,3050,1697,1635,820$. EIMS $\mathrm{m} / z$ (rel. int.): $[\mathrm{M}]^{+}$ 456 (10), 411 (22), 248 (34), 203 (100), 189 (16). HREIMS m/z: 456.3599 (calcd. for $\mathrm{C}_{30} \mathrm{H}_{48} \mathrm{O}_{3}, 456.3603$ ). ${ }^{1} \mathrm{H}$ NMR $\left(\mathrm{CDCl}_{3}, 400 \mathrm{MHz}\right) \delta: 5.11(1 \mathrm{H}, \mathrm{m}, \mathrm{H}-12), 3.19(1 \mathrm{H}, \mathrm{dd} J=$ $10.0 \mathrm{~Hz}, J=4.5 \mathrm{~Hz}), 1.20(3 \mathrm{H}, \mathrm{s}, \mathrm{Me}-27), 1.07$ (3H, s, Me23), 0.94 ( $3 \mathrm{H}, \mathrm{s}, \mathrm{Me}-25), 0.91$ ( $3 \mathrm{H}, \mathrm{d}, J=6.6 \mathrm{~Hz}, \mathrm{Me}-30)$, 0.86 (3H, s, Me-26), 0.81 (3H, s, Me-24), 0.80 (3H, d, $J=6.8$ $\mathrm{Hz}, \mathrm{Me}-29) .{ }^{13} \mathrm{C} \mathrm{NMR}\left(\mathrm{CDCl}_{3}, 100 \mathrm{MHz}\right) \delta: 176.2$ (C-28), 138.7 (C-13), 125.8 (C-12), 79.1 (C-3), 55.2 (C-18), 52.4 (C-5), 48.9 (C-17), 47.4 (C-9), 42.0 (C-14), 39.6 (C-8), 38.5 (C-1), 37.0 (C-22), 36.1 (C-10), 33.2 (C-7), 30.5 (C-19), 32.3 (C-20), 29.4 (C-15), 27.5 (C-21), 24.6 (C-27), 24.5 (C-16), 27.4 (C2), 24.0 (C-23) 23.9 (C-11), 23.8 (C-30), 22.4 (C-29), 18.3 (C-6), 17.6 (C-26), 15.7 (C-25) 15.4 (C-24).

Cyclostenol (13): Colorless crystals, 28 mg, m.p. 221$222{ }^{\circ} \mathrm{C} .[\alpha]_{\mathrm{D}}{ }^{30}+43.2^{\circ}\left(\mathrm{c}=1.50, \mathrm{CHCl}_{3}\right)$. IR $\left(\mathrm{KBr}, \mathrm{v}_{\max }, \mathrm{cm}^{-1}\right)$ : 3435-3380 (OH), 1360-1380 (germinal dimethyl). EIMS $\mathrm{m} / \mathrm{z}$ (rel. int.): $[\mathrm{M}]^{+} 416$ (35), 398 (48), 383 (100), 338 (30). HREIMS $m / z$ : 416.5210 (calcd. 416.5236 for $\mathrm{C}_{28} \mathrm{H}_{48} \mathrm{O}_{2}$ ). ${ }^{1} \mathrm{H}$ NMR $\left(\mathrm{CDCl}_{3}, 400 \mathrm{MHz}\right) \delta: 3.57(1 \mathrm{H}, \mathrm{dd}, J=10.4,4.9 \mathrm{~Hz}$, H-3), 3.22 (1H, dt, $J=9.9,7.6 \mathrm{~Hz}, \mathrm{H}-6), 1.62(2 \mathrm{H}), 1.06(2 \mathrm{H})$, $0.52(2 \mathrm{H}, \mathrm{d}, J=4.7 \mathrm{~Hz}, \mathrm{H}-19), 0.97$ (3H, s, H-28), $0.92(3 \mathrm{H}$, $\mathrm{d}, J=7.3 \mathrm{~Hz}, \mathrm{H}-21), 0.87$ (3H, s, H-18), $0.83(6 \mathrm{H}, \mathrm{d}, J=6.8$ $\mathrm{Hz}, \mathrm{H}-26$ and $\mathrm{H}-27) .{ }^{13} \mathrm{C} \mathrm{NMR}\left(\mathrm{CDCl}_{3}, 100 \mathrm{MHz}\right) \delta: 70.7$ (C-6), 70.2 (C-3), 52.3 (C-17), 47.4 (C-14), 47.3 (C-8), 39.4 (C-24), 26.3 (C-11), 36.0 (C-22), 34.4 (C-12), 32.2 (C-15), 30.7 (C-1), 28.0 (C-25), 27.0 (C-16), 29.2 (C-10), 25.0 (C-19), 24.0 (C-23), 23.1 (C-26, 27), 18.8 (C-21), 18.7 (C-28), 17.8 (C-18).

Oleanolic acid (14): Colorless crystals, $22 \mathrm{mg}$, m.p. 305$306^{\circ} \mathrm{C} .[\alpha]_{\mathrm{D}}^{25}+78.9^{\circ}\left(\mathrm{c}=0.07, \mathrm{CHCl}_{3}\right)$. IR $\left(\mathrm{KBr}, \nu_{\max }, \mathrm{cm}^{-1}\right)$ : 3400-2640, 1700, 1660 and 820. EIMS $m / z$ (rel. int.): 456 $\left[\mathrm{M}^{+}(4), 248\right.$ (98), 208 (12), 203 (60) and 133 (53). HREIMS $m / z: 456.3610$ (calcd. for $\mathrm{C}_{30} \mathrm{H}_{48} \mathrm{O}_{3}, 456.3603$ ). ${ }^{1} \mathrm{H}$ NMR $\left(\mathrm{CDCl}_{3}, 400 \mathrm{MHz}\right): \delta 5.24(1 \mathrm{H}, \mathrm{t}, J=3.4 \mathrm{~Hz}, \mathrm{H}-12), 3.60(1 \mathrm{H}$, dd, $J=4.1,9.9 \mathrm{~Hz}, \mathrm{H}-3), 1.12$ (3H, s, Me-27), 1.03 (3H, s, Me-23), 0.98 (3H, s, Me-24), 0.96 (3H, s, Me-25), 0.91(3H, s, Me-29), 0.90 (3H, s, Me-26) and 0.89 (3H, s, Me-30). ${ }^{13} \mathrm{C}$ NMR $\left(\mathrm{CDCl}_{3}, 100 \mathrm{MHz}\right): \delta 183.4(\mathrm{C}-28), 143.6(\mathrm{C}-13), 122.7$ (C-12), 78.0 (C-3), 55.2 (C-5), 47.6 (C-9), 46.5 (C-17), 45.9 (C-19), 41.6 (C-14), 41.0 (C-18), 39.1 (C-8), 38.9 (C-4), 38.4 (C-1), 37.5 (C-10), 33.8 (C-21), 33.0 (C-29), 32.2 (C-7), 32.4 (C-22), 30.6 (C-20), 28.1 (C-23), 27.5 (C-15), 27.2 (C-2), 25.9 
(C-27), 24.5 (C-30), 23.4 (C-11), 23.4 (C-16), 19.3 (C-6), 17.1

(C-26), 15.6 (C-24), 15.4 (C-25).

5, 6-Dihydroxy-4,7-dimethoxy-2-methylanthracene9,10-dione (15): Orange crystals, m.p. $259^{\circ} \mathrm{C}$. UV (MeOH): $\lambda_{\max } 306,329,405 \mathrm{~nm}$. IR (KBr, $\left.v_{\max }, \mathrm{cm}^{-1}\right) 3355,1659,1635$, 1455. EIMS $m / z$ (rel. int. \%) $\left[\mathrm{M}^{+} 314\right.$ (66), 299 (29), 296 (35), 283 (19), 269 (30), 268 (40), 255 (15). HREIMS m/z (rel. int. \%) 314.0785 (calcd. for $\left.\mathrm{C}_{17} \mathrm{H}_{14} \mathrm{O}_{6}, 314.0790\right) .{ }^{1} \mathrm{H}$ NMR $\left(\mathrm{CDCl}_{3}, 400 \mathrm{MHz}\right) \delta: 12.01(1 \mathrm{H}, \mathrm{s}, \mathrm{OH}-5), 7.60(1 \mathrm{H}, \mathrm{s}, \mathrm{H}-1)$, 7.23 (1H, s, H-8), 7.09 (1H, s, H-3), 6.50 (1H, s, OH-6), 3.95 $\left(3 \mathrm{H}, \mathrm{s}, \mathrm{OCH}_{3}-7\right), 3.89$ (3H, s, $\left.\mathrm{OCH}_{3}-8\right), 2.49\left(3 \mathrm{H}, \mathrm{s}, \mathrm{CH}_{3}-2\right)$. ${ }^{13} \mathrm{C} \mathrm{NMR}\left(\mathrm{CDCl}_{3}, 100 \mathrm{MHz}\right) \delta: 185.0$ (C-10), 181.0 (C-9), 160.5 (C-4), 154.1 (C-5), 150.9 (C-7), 148.9 (C-2), 139.1 (C6), 123.0 (C-3), 120.9 (C-1), 106.5 (C-8), $63.3\left(\mathrm{OCH}_{3}-4\right), 56.0$ $\left(\mathrm{OCH}_{3}-7\right), 22.0\left(\mathrm{CH}_{3}-2\right)$.

Cycloart-24-ene-1,3-diol (16): White crystals, $16 \mathrm{mg}$, m.p. $140.5^{\circ} \mathrm{C},[\alpha]_{\mathrm{D}}{ }^{25}+78.9^{\circ}\left(\mathrm{c}=0.07, \mathrm{CHCl}_{3}\right)$. IR (KBr, $v_{\max }$, $\mathrm{cm}^{-1}$ ) 3440-3380 (OH), 1360-1380 (geminaldimethyls). EIMS (rel. int. \%) [M] 442 (26), 424 (66), 406 (52), 391 (45), 346 (35). HREIMS (rel. int. \%) [M] 442 (calcd. 442.2413 for $\left.\mathrm{C}_{30} \mathrm{H}_{50} \mathrm{O}_{2}\right),{ }^{1} \mathrm{H} \mathrm{NMR}\left(\mathrm{CDCl}_{3}, 400 \mathrm{MHz}\right) \delta: 5.10(1 \mathrm{H}, \mathrm{t}, J=6.2$ $\mathrm{Hz}, \mathrm{H}-24), 3.22$ (1H, dd, $J=6.9,4.4 \mathrm{~Hz}, \mathrm{H}-1), 3.31$ (1H, dd, $J=9.2,6.8 \mathrm{~Hz}, \mathrm{H}-3), 1.54$ (6H, s, Me-26, Me-27), $1.15(3 \mathrm{H}$, s, Me-18), 1.14 (3H, s, Me-28), 1.12 (3H, s, Me-30), 1.09 (3H, s, Me-29), 1.03 (3H, s, Me-21), $0.06(2 \mathrm{H}, \mathrm{d}, J=4.4 \mathrm{~Hz}$, $\mathrm{H}-18) .{ }^{13} \mathrm{C} \mathrm{NMR}\left(\mathrm{CDCl}_{3}, 100 \mathrm{MHz}\right), 130.0$ (C-25), 124.1 (C-24), 76.2 (C-3), 54.3 (C-13), 53.1 (C-1), 51.0 (C-5), 50.9 (C-8), 46.0 (C-17), 41.4 (C-15), 39.5 (C-14), 37.4 (C-20), 36.9 (C-22), 36.2 (C-4), 34.5 (C-11), 30.2 (C-10), 28.6 (C-19), 27.3 (C-16), 25.9 (C-23), 24.9 (C-9), 22.6 (C-28), 21.8 (C-6), 21.8 (C-26), 20.7 (C-12), 20.6 (C-29),18.0 (C-30), 17.1 (C-27), $16.9(\mathrm{C}-21)$

Stigmasterol 3-O $\boldsymbol{\beta} \boldsymbol{\beta}$-D-glucopyranoside (17): Amorphous solid, $30 \mathrm{mg}$, m.p. $289-290^{\circ} \mathrm{C}$. $[\alpha]_{\mathrm{D}}{ }^{27}-51.5^{\circ}\left(\mathrm{c}=1.20, \mathrm{CH}_{3} \mathrm{OH}\right)$. IR $\left(\mathrm{KBr}, \nu_{\max }, \mathrm{cm}^{-1}\right) 3454(\mathrm{OH}), 3024,1646(\mathrm{C}=\mathrm{C})$. EIMS m/z: [M-Glc] $^{+} 412$ (72), 397 (15), 394 (22), 379 (28), 369 (35), 351 (71), 327 (55), 301(15), 300 (67), 273 (21), 271 (26). HRFABMS (+ve) $\mathrm{m} / z$ : 575.4231 (calcd. for $\mathrm{C}_{35} \mathrm{H}_{59} \mathrm{O}_{6}$, 575.4233). ${ }^{1} \mathrm{H}$ NMR (400 MHz, $\left.\mathrm{CD}_{3} \mathrm{OD}\right) \delta: 5.23(1 \mathrm{H}$, br d, $J=5.4 \mathrm{~Hz}, \mathrm{H}-6), 5.14$ (1H, dd, $J=15.2,8.4 \mathrm{~Hz}, \mathrm{H}-22), 5.02$ $(1 \mathrm{H}, \mathrm{dd}, J=15.2,8.6 \mathrm{~Hz}, \mathrm{H}-23), 4.78(1 \mathrm{H}, \mathrm{d}, J=7.4 \mathrm{~Hz}, \mathrm{H}-$ 1'), 3.84-4.44 (m, Glc-H), 3.83 (1H, m, H-3), 1.01 (3H, s, $\left.\mathrm{CH}_{3}-19\right), 0.90$ (3H, d, $\left.J=6.2 \mathrm{~Hz}, \mathrm{CH}_{3}-21\right), 0.83$ (3H, d, $J=$ $\left.6.5 \mathrm{~Hz}, \mathrm{CH}_{3}-26\right), 0.82\left(3 \mathrm{H}, \mathrm{t}, J=7.0 \mathrm{~Hz}, \mathrm{CH}_{3}-29\right), 0.80(3 \mathrm{H}$, $\left.\mathrm{d}, J=6.5 \mathrm{~Hz}, \mathrm{CH}_{3}-27\right), 0.67$ (3H, s, $\left.\mathrm{CH}_{3}-18\right) .{ }^{13} \mathrm{C}$ NMR $(125$ $\left.\mathrm{MHz}, \mathrm{CD}_{3} \mathrm{OD}\right) \delta: 141.5$ (C-5), 138.9 (C-22), 129.1 (C-23), 121.1 (C-6), 102.8 (C-1'), 79.8 (C-3), 76.9 (C-3'), 76.7 (C5'), 74.2 (C-2'), 70.6 (C-4'), 62.2 (C-6'), 57.0 (C-14), 56.1 (C-17), 52.1 (C-24), 50.8 (C-9), 43.9 (C-4), 43.1 (C-13), 40.5 (C-20), 39.9 (C-12), 37.8 (C-1), 36.9 (C-10), 32.9 (C-25), 32.8 (C-2), 31.9 (C-7), 31.7 (C-8), 28.9 (C-16), 25.6 (C-28), 24.5 (C-15), 21.9 (C-21), 21.7 (C-27), 21.5 (C-11), 19.5 (C-19), 19.1 (C-26), 12.6 (C-18), 12.1 (C-29).

$\boldsymbol{\beta}$-Sitosterol 3- $\boldsymbol{O}$ - $\boldsymbol{\beta}$-D-glucopyranoside (18): Amorphous solid, $18 \mathrm{mg}$, m.p. $279-280{ }^{\circ} \mathrm{C},[\alpha]_{\mathrm{D}}{ }^{28}-14.5^{\circ}(\mathrm{c}=1.32, \mathrm{MeOH})$. IR $\left(\mathrm{KBr}, v_{\max }, \mathrm{cm}^{-1}\right) 3452,3044,1646$. EIMS m/z: [M-Glc] $]^{+}$ 414 (19), 399 (15), 396 (24), 381 (70) 329 (25), 275 (11), 273 (24), 255 (35). HRFABMS (+ve) $m / z: 577.4386[\mathrm{M}+\mathrm{H}]^{+}$ (calcd. for $\left.\mathrm{C}_{35} \mathrm{H}_{61} \mathrm{O}_{6}, 577.4389\right)$. ${ }^{1} \mathrm{H}$ NMR (300 MHz, $\mathrm{CD}_{3} \mathrm{OD}$ ) $\delta: 5.33\left(1 \mathrm{H}, \mathrm{d}, J=7.2 \mathrm{~Hz}, \mathrm{H}-1^{\prime}\right), 5.12(1 \mathrm{H}, \mathrm{br} \mathrm{d}, J=5.4 \mathrm{~Hz}$, H-6), 3.85 (1H, m, H-3), 3.82-4.42 (m, Glc-H), 1.01 (3H, s, $\left.\mathrm{CH}_{3}-19\right), 0.92\left(3 \mathrm{H}, \mathrm{d}, J=6.2 \mathrm{~Hz}, \mathrm{CH}_{3}-21\right), 0.84$ (3H, t, $J=7.0$ $\left.\mathrm{Hz}, \mathrm{CH}_{3}-29\right), 0.83$ (3H, d, $\left.J=6.5 \mathrm{~Hz}, \mathrm{CH}_{3}-26\right), 0.81$ (3H, d, $J$ $\left.=6.5 \mathrm{~Hz}, \mathrm{CH}_{3}-27\right), 0.68\left(3 \mathrm{H}, \mathrm{s}, \mathrm{CH}_{3}-18\right) .13 \mathrm{C}-\mathrm{NMR}(100 \mathrm{MHz}$, $\left.\mathrm{CD}_{3} \mathrm{OD}\right) \delta$ : 142.0 (C-5), 122.1 (C-6), $103.2\left(\mathrm{C}-1^{\prime}\right), 80.9$ (C3), $76.8\left(\mathrm{C}-3^{\prime}\right), 76.6\left(\mathrm{C}-5^{\prime}\right), 74.2\left(\mathrm{C}-2^{\prime}\right), 70.2\left(\mathrm{C}-4^{\prime}\right), 62.0\left(\mathrm{C}-6^{\prime}\right)$, 56.9 (C-14), 56.5 (C-17), 50.5 (C-24), 50.4 (C-9), 43.9 (C-4), 43.0 (C-13), 40.8 (C-12), 39.5 (C-22), 38.7 (C-1), 37.2 (C-20), 37.1 (C-10), 33.0 (C-7), 32.9 (C-8), 29.9 (C-2), 29.7 (C-16), 29.5 (C-23), 26.0 (C-25), 25.8 (C-15), 23.7 (C-28), 21.5 (C-11), 20.1 (C-27), 19.7 (C-21), 19.5 (C-19), 18.2 (C-26), 12.2 (C-18), 11.9 (C-29).

\section{RESULTS AND DISCUSSION}

The methanolic extract of Tribulus longipetalus was divided into $n$-hexane, chloroform, ethyl acetate, $n$-butanol and water soluble fractions. A series of column chromatographic techniques applied to chloroform and ethyl acetate fractions resulted in the isolation and structural elucidation of eighteen known compounds and were identified as 1-triacontanol ${ }^{4}(\mathbf{1})$, hexacosanoic $\operatorname{acid}^{5}(2)$, tetracosanoicacid ${ }^{6}(3)$, benzoic $\operatorname{acid}^{7}(4)$, 2-hydroxybenzoic $\operatorname{acid}^{8}(5), 4$-ethoxy benzoic $\operatorname{acid}^{9}(\mathbf{6}), 3,5-$ dimethoxybenzoic $\operatorname{acid}^{10}(7), \beta$-sitosterol ${ }^{4}(8), \beta$-amyrin ${ }^{11}(\mathbf{9})$, stigmasterol $^{12}(\mathbf{1 0}), 5 \alpha, 8 \alpha$-epidioxyergosta-6, $9(11), 22$ - trien$3 \beta-\mathrm{ol}^{13}(\mathbf{1 1})$, ursolic acid $^{4,10}(\mathbf{1 2})$, cyclostenol ${ }^{14}(\mathbf{1 3})$, olea-nolic $\operatorname{acid}^{15}(\mathbf{1 5}), 5,6$-dihydroxy-4, 7-dimethoxy-2-methy-lanthracene9,10-dione ${ }^{16}$ (15), 1,3-dihydroxycyclolanosterol ${ }^{17}$ (16), stigmasterol 3-O- $\beta$-D-glucopyranoside ${ }^{15}(\mathbf{1 7}), \beta$-sitosterol 3O- $\beta$-D-glucopyranoside ${ }^{18}(\mathbf{1 8})$, respectively. All of these compounds have been purified for the first time from Tribulus longipetalus Viv.

\section{REFERENCES}

1. A. Gafoor, Flora of West Pakistan, Printas Printing Press, Karachi, Pakistan, p. 21 (1974).

2. S.R. Baquar, Medicinal And Poisonous Plants of Pakistan, Printas Printing Press, Karachi, Pakistan, p. 451 (1989).

3. A. Temraz, D.O. El Gindi, H.A. Kadry, N. De Tommasi and A. Braca, Phytochemistry, 67, 1011 (2006).

4. E. Ahmed, A. Malik, N. Riaz and A. Sharif, J. Chem. Soc. Pak., 26, 389 (2004)

5. S. Watanabe, Z. Naturforsch. C, 30, 825 (1975).

6. L. Marosi, Annalen, 584 (1973)

7. E. Ahmed, A. Malik, M.M. Ahmad, M.A. Munawar, S.A. Nagar, J. Anwar, M.A. Qazi, A. Sharif, N. Afza and M. Ashraf, J. Chem. Soc. Pak., 30, 936 (2008).

8. Aldrich Library of ${ }^{13} \mathrm{C}$-and ${ }^{1} \mathrm{H}-\mathrm{FT}-\mathrm{NMR}$ Spectra, 2, 1065B, 1065C, 1251B, 1252A, 1414C, 1511A, 1512A (1992).

9. K.N. Scott, J. Am. Chem. Soc., 94, 8564 (1972).

10. K. Jabeen, A. Javaid, E. Ahmad and M.M. Athar, Nat. Prod. Res., 25, 264 (2011)

11. S. Hussain, E. Ahmed, A. Malik and A. Jabbar, J. Chem. Soc. Pak., 27, 296 (2005).

12. H.L. Holland, P.R.P. Diakow and G.J. Taylor, Can. J. Chem., 56, 3121 (1978).

13. A.A.C. Gunatilaka, Y. Gopichand, F.J. Schmitz and C. Djerassi, J. Org. Chem., 46, 3860 (1981).

14. H.W. Kircher and H.L. Bird Jr., Phytochemistry, 21, 1705 (1982).

15. E. Ahmed, A. Sharif, S. Hussain, A. Malik and M. Ul Hassan, J. Chem. Soc. Pak., 33, 676 (2011).

16. A.G. Gonzalez, J.B. Barrera, B.B. Davila, E. Valencia and X.A. Dominguez, Phytochemistry, 31, 255 (1992).

17. T. Shen, H.Q. Yuan, W.Z. Wan, X.L. Wang, X.N. Wang, M. Ji and H.X. Lou, J. Nat. Prod., 71, 81 (2008).

18. E. Ahmed, A. Sharif, H. Nawaz and M. Ul Hassan, J. Chem. Soc. Pak., 33, 682 (2011). 